\title{
Benefits of an Active Spine Supported Bounding Locomotion With a Small Compliant Quadruped Robot
}

\author{
M. Khoramshahi ${ }^{\dagger}, \#$, A. Spröwitz ${ }^{\dagger}$, A. Tuleu ${ }^{\dagger}$, M. N. Ahmadabadi $\#$, A. J. Ijspeert ${ }^{\dagger}$
}

\begin{abstract}
We studied the effect of the control of an active spine versus a fixed spine, on a quadruped robot running in bound gait. Active spine supported actuation led to faster locomotion, with less foot sliding on the ground, and a higher stability to go straight forward. However, we did no observe an improvement of cost of transport of the spine-actuated, faster robot system compared to the rigid spine.
\end{abstract}

\section{INTRODUCTION}

Recently, research in quadruped locomotion has shifted away from statically stable locomotion, e.g. with the well researched Aibo robot [1], towards faster and more dynamic locomotion.

Quadruped robots now mimic gaits found by quadruped animals, such as walk-Tekken1 [2], Cheetah [3], paceRaibert's quadruped [4], trot-Raibert's Quadruped [4], Tekken1 [2], HyQ [5], and bound-Raiberts' Quadruped [4], Tekken1 [2], Puppy1 [6], ScoutII [7], Puppy2 [8], BigDog [9], Rush [10], PAW [11].

Bound is an in-phase gait [12] like gallop and half-bound, however with a symmetry between left and right legs. A corresponding gait diagram is given in Fig. 11b. In terms of speed, bounding gaits are typically faster than walk, trot, and pace, but slower than gallop. For locomotion, bounding animals not only make use of their leg muscles and joints, but also of their spine. [12, p. 1337] report that: "[...] the main part of body propulsion - half the step length - is contributed by additive spine movements". Until recently, quadruped robots however have only sparsely made use of active or passive spine movements [13]. An extremely fast running robot applying spine movement, reported maximum speed is of more than $12.5 \mathrm{~m} \mathrm{~s}^{-1}$, is the currently developed "Boston Dynamics Cheetah robot" [14]. However, no scientific data is available on it.

We report on experiments with Bobcat-robot, a small RCservo motor controlled quadruped robot, with compliant twosegment legs, and one active spine joint. A spine-mounted RC servo motor allows for intra-trunk rotations, in the sagittal plane. We defined the following two hypotheses, and tested them on our robotic platform: (1) active spine movement increases maximum possible running (bounding) speed; (2) higher spine amplitude contributes directly to higher robot speed. Fig. 2 illustrates these hypotheses, in particular that a higher robot speed can be expected with a spine oscillation upwards and downwards from the horizontal line, eventually increasing foot distance (potentially: step length).

In this work, we report on results with the robot hardware, for 222 individual experimental runs, on the resulting robot

†Biorobotics Laboratory, Institute of Bioengineering, EPFL, Switzerland. \#Robotics and AI Lab., Control and Intelligent Processing Center of Excellence, ECE Department, University of Tehran, Iran; corresponding author: alexander.sproewitz@epfl.ch

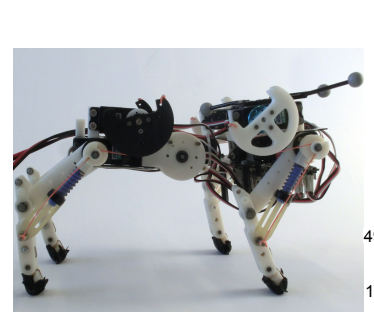

(a)

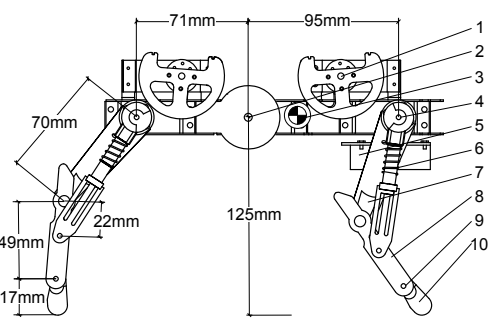

(b)
Fig. 1: Left: Sideview of Bobcat-robot, the front is to the right side. Two mounted, reflective spheres of the motion capture system are visible on the top right. Red cables along the legs connect knee servo motors (1) and knee joints, and compress the robot's legs. During swing phase, this ensures foot clearance, during stance phase this stabilizes the vertical position of the corresponding proximal joint. Right: Side view at CAD presentation of Bobcat-robot. (1) knee RC servo motor, (2) spine RC servo motor, (3) robot center of mass (COM), (4) hip RC servo motor, (5) RB110 control board, (6) compression, linear leg spring, (7) upper leg segment, (8) lower leg segment, (9) rotatory, linear foot spring (hidden), (10) foot segment.

speed for four different gait categories (fixed spine, small amplitude spine, high amplitude spine, and high voltageboosted high amplitude spine), the cost of transport (COT) of all reported gaits $\left(\mathrm{COT}=\frac{P_{\text {elec }}}{\mathrm{mgv}}\right.$, dimensionless: $[15],[16]$, or e.g. $\left.\left[\mathrm{JN}^{-1} \mathrm{~m}^{-1}\right]\right)$, and the range of robot pitching motion (rotation in sagittal plane).

\section{HARDWARE AND EXPERIMENTS}

This section explains firstly the hardware and experimental setup, by briefly describing characteristics of the quadruped robot, its control framework, and the experimental setup. The description of the experiments follows.

1) Robot Mechanics: Bobcat-robot (Fig. 1a) was previously developed at the Biorobotics Laboratory (EPFL, Switzerland), here we apply it as a rapid testing tool for different spine actuation scenarios. It includes nine active degrees of freedom (DOF, two per leg, one at the spine), with RC servo motors as actuators (Kondo KRS 2350 ICS, stall torque $2 \mathrm{Nm}$ at $6 \mathrm{~V}$ ). The robot is build from $3 \mathrm{~d}$ printed ABS plastic, POM, and sheet carbon and glass fiber materials.

Bobcat-robot legs feature two main segments (upper and lower), and a short foot segment. Front and hind legs are identical. "Knees" are oriented backwards, similarly to Puppy2 robot [6]. A linear compression spring (Fig. 1b, number 6) extends the robot's legs against gravity. Robot hip height is $0.125 \mathrm{~mm}(0.11 \mathrm{~mm})$, for non-stressed (stressed, standing height) leg springs. During locomotion, leg length was reduced up to $90 \mathrm{~mm}$. Legs are actively compressed ("knee actuation") by activating the knee RC servo motors, which are mounted 


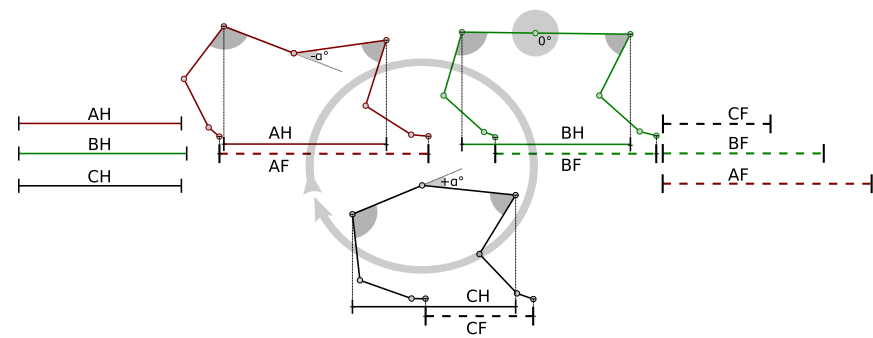

Fig. 2: Schematic presentation of spine movements for one possible spine joint configuration, with a near-center, single rotational active joint between hip and shoulder joints. Broken lines present distance between front and hind proximal joint, solid horizontal lines distance between hip and shoulder. All hip/shoulder angles are kept constant. Through this, spine angular rotation is multiplying distance between front and hind foot largely, while distance between hip and shoulder point changes in comparison minimally. Flight phases, hip joint, and knee joint movements are omitted for simplification. AH: case A (spine bent downwards, distance between hips), AF: case A, distance between feet, $\mathrm{BH}$ : horizontal spine, distance between hips, BF: case B, feet-distance, $\mathrm{CH}$ and $\mathrm{CF}$ accordingly.

proximally. Knee actuators are connected to the robot's knees by a flexible cable. Mounting the knee motor proximally, and applying above cable mechanism was implemented to decouple hip and knee actuation, while keeping leg inertia low. The cable mechanism acts as an automatic clutch, in case external forces exceed knee actuator forces.

The robot's spine actuation is applying a single RC servo motor, positioned $71 \mathrm{~mm}$ (/95 $\mathrm{mm}$ ) from the hip (/shoulder) joint (Fig. 1b). Including mechanical limits, the range of spine movement is 35 degree upwards and downwards from the horizontal. For one set of experiments (fixed spine, Tab. II) we locked the spine joint mechanically, with fixation pins. Bobcat-robot's center of mass (COM) is roughly at the height of its hip/shoulder joints, $78 \mathrm{~mm}$ behind the shoulder joints.

Bobcat-robot's front legs are slightly more far apart (sideways direction, $127 \mathrm{~mm}$ ) than its hind legs $(97 \mathrm{~mm})$, to avoid potential leg collisions between front and hind legs. After a series of first tests, the robot was equipped with short additional "foot" segments. Those are coupled by a set of rotational springs to the lower leg segments. Spring coupled feet improved ground traction significantly. We applied Duct tape to cover the robot's feet, and replaced the tape layer regularity.

The robot weights $1.1 \mathrm{~kg}$, including $\mathrm{RC}$ servo motors, mechanics, and a RB110, single-board computer control board. For experimental convenience, we used a power tether, supplying electrical power. Central pattern generator (CPG) parameters were transmitted via a wireless communication module plugged to the RB110 control board. The CPG's oscillators ran online on the RB110 control board, with a fixed Newton-Euler integration time step of $5 \mathrm{~ms}$. CPG outputs were interpreted as position signals (motor command) to create pulse width modulation (PWM) patterns (frequency $50 \mathrm{~Hz}$ ) controlling the robot's RC servo motors.

2) $C P G$ and control: We implemented a computational Central Pattern Generator (CPG), as a network of nine coupled nonlinear oscillators (two per leg, one for the spine). An earlier implementation this CPG setup (configuration legonly) can be found in [17], for a small quadruped robot
TABLE I: Bobcat-robot characteristics.

$\begin{array}{lrr}\text { Leg weight (fore/hind leg) } & 33 & \mathrm{~g} \\ \text { Total weight } & 1031 & \mathrm{~g} \\ \text { Touchdown leg length (fore/hind leg) } & 125 & \mathrm{~mm} \\ \text { Robot length } & 166 & \mathrm{~mm} \\ \text { Robot width (fore/hind) } & 127 / 97 & \mathrm{~mm}\end{array}$

running in trot gait. Although potentially possible, we did not implement feedback in this work, but we currently run all experiments with open-loop control patterns, acting in the robot's joint space (opposed to end effector space). Each oscillator presents one active degree of freedom. Phase couplings between hip oscillators $\left(k_{i, j}\right.$ for connectivity matrix, $\varphi_{i, j}$ for phase lag values) determine the gait of the quadruped, here bounding. A fully connected connectivity graph of the oscillator network is given in Fig. 3a. Knee/spine and hip os-
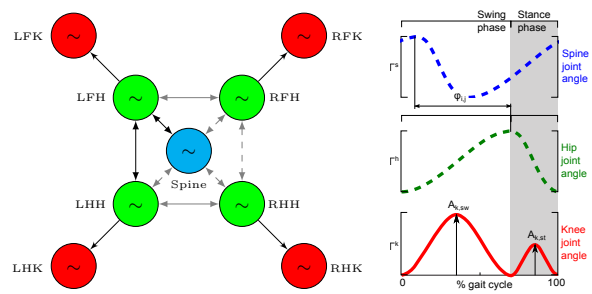

Fig. 3: Left figure (a): Fully connected CPG network, with nine oscillator nodes. LFK: left front knee oscillator, LHH: left hind hip oscillator and so on. Black couplings represent open parameter, grey couplings use a phase lag of 0 , dashed grey couplings are calculated to produce a cirlce phase lag value of zero $\bmod 2 \pi$. Right figure (b): Spine, hip, and knee position signal produced by their corresponding oscillators. The global maximum signal of the knee oscillator corresponds to a large leg length reduction during swing phase, for foot clearance. The second smaller, local maximum occurs during mid-stance, and adapts stance leg length.

cillators produced two patterns (Fig. 3b), due to an additional knee filter set in-series to the knee oscillators phase signal. Amplitudes $(A)$, offset values $(O)$, and phase couplings $(\phi)$, are directly controlled through their corresponding CPG parameters. $\alpha$ is a fixed gain parameter. Hip oscillator setpoint $\left(\Theta_{i}^{h}\right)$ is calculated as follows, whereas the spine joint $\left(\Theta_{i}^{s}\right)$ is treated as a "computational 5th hip joint", with individual values for offset, amplitude, and phase shift:

$$
\begin{aligned}
\dot{\phi}_{i} & =2 \pi f+\sum_{j \neq i} k_{i, j} \sin \left(\phi_{j}-\phi_{i}-\varphi_{i, j}\right) \\
\dot{a}_{i}^{h, s} & =\alpha\left(A_{i}^{h, s}-a_{i}^{h, s}\right) \\
\dot{o}_{i}^{h, s} & =\alpha\left(O_{i}^{h, s}-o_{i}^{h, s}\right) \\
\Theta_{i}^{h, s} & = \begin{cases}\frac{\phi_{i}}{2 D_{\mathrm{vir}}} & 0 \leq \phi_{i} \leq 2 \pi D_{\mathrm{vir}} \\
\frac{\phi_{i}+2 \pi\left(1-2 D_{\mathrm{vir}}\right)}{2\left(1-D_{\mathrm{vir}}\right)} & \end{cases}
\end{aligned}
$$

Virtual duty factor $D_{\text {vir }}$ is the ratio between leg retraction duration and the complete stride cycle duration (joint space). $D_{\text {vir }}$ is a control parameter, it is to be distinguished from the real duty factor $D$, which in animal gaits describes the value measured through foot fall (task space) patterns [18]. Hip/spine joint motor command $\Gamma_{h}$ is derived as:

$$
\Gamma_{i}=a_{i}^{h, s} \cos \left(\Theta_{i}^{h, s}\right)+o_{i}^{h, s}
$$


During swing phase the controller actively reduces leg length, to avoid collision between the swinging foot and the ground. During mid stance phase, knee stance-phase deflection can shorten leg length. This parameter aims to decrease vertical hip oscillations of the stance leg. Knee deflections (swingphase: $A_{\mathrm{sw}}^{\mathrm{k}}$, stance-phase: $\left.A_{\mathrm{st}}^{\mathrm{k}}\right)$ are defined unit-less, as a fraction of leg length. Knee phase is set as:

$$
\Theta_{i}^{k}=\Theta_{i}^{h}+\varphi_{h, k}
$$

The knee joint motor receives $\Gamma_{k}$ as follows, the knee joint profile adjustment is made using a piecewise cubic profile:

$$
\begin{aligned}
a_{i}^{k} & =\left\{\begin{array}{c}
A_{s t}^{k}, \text { if } \Theta_{i}^{k}<\pi \\
A_{s w}^{k}, \text { if } \Theta_{i}^{k} \geq \pi
\end{array}\right. \\
\dot{o}_{i}^{k} & =\alpha\left(O_{i}^{k}-o_{i}^{k}\right) \\
\theta_{i}^{\prime} & \equiv \frac{\Theta_{i}^{k}}{2 \pi}(\bmod 0.5) \\
\theta_{i} & =2 \cdot \theta_{i}^{\prime} \\
\gamma_{i} & =\left\{\begin{array}{l}
-16 \theta_{i}^{3}+12 \theta_{i}^{2}, \text { if } \theta_{i}<0.5 \\
12\left(\theta_{i}-0.5\right)^{3}-12\left(\theta_{i}-0.5\right)^{2}+1
\end{array}\right. \\
\Gamma_{i}^{k} & =a_{k}^{i} \gamma_{i}+o_{i}^{k}
\end{aligned}
$$

The resulting open-loop trajectories are plotted for one stride cycle (Fig. 3b).

TABLE II: Four gait categories applied in this work. Voltage was increased largely to ensure good amplitude and speed tracking, also at higher RC-servo motor oscillations. This reduced continuous experimental run time, RC-servo motors heated faster, and sufficient cool-down times were necessary. Typically, in high-voltage range, 4-5 min of continuous experiments were possible. Given speed values are maximum, average values per gait category.

\begin{tabular}{lccccc} 
Gait category & $\begin{array}{c}\text { Number } \\
\text { of gaits }\end{array}$ & $\begin{array}{c}A_{\text {spine }}[\mathrm{deg}] \\
{[\mathrm{V}]}\end{array}$ & $\begin{array}{c}\text { Volt. } \\
{[\mathrm{Speed}} \\
{\left[\mathrm{m} \mathrm{s}^{-1}\right]}\end{array}$ & $\begin{array}{c}\text { COT } \\
{[\mathrm{J} / \mathrm{Nm}]}\end{array}$ \\
\hline Rigid spine & 80 & 0 & 8,9 & 0.62 & 6.5 \\
Small sp. amp. & 80 & 15 & 8,9 & 0.71 & 6.3 \\
High sp. amp. & 50 & 30 & 8,9 & 0.67 & 7.4 \\
High voltage & 12 & 30 & 12 & 0.78 & 10.9
\end{tabular}

TABLE III: CPG parameters of the best gait, from the two experimental runs with the rigid spine, and the active spine.

\begin{tabular}{llrr} 
CPG Para. & & Rigid spine gait & Active spine gait \\
\hline$f$ & {$[\mathrm{~Hz}]$} & 3.5 & 4 \\
$A_{\text {hip }}^{\text {fore }}$ & {$[\mathrm{rad}]$} & 0.875 & 0.5 \\
$A_{\text {hind }}^{\text {hind }}$ & {$[\mathrm{rad}]$} & 0.875 & 0.4 \\
$D_{\text {vir }}$ & {[]} & 0.48 & 0.5 \\
$O_{\text {hip }}^{\text {fore }}$ & {$[\mathrm{rad}]$} & -0.3 & 0.15 \\
$O_{\text {hind }}^{\text {hind }}$ & {$[\mathrm{rad}]$} & -0.32 & -0.4 \\
$\phi_{\text {hip }}$ & {$[\mathrm{rad}]$} & 2.8 & 3.14 \\
$A_{\text {spine }}$ & {$[\mathrm{rad}]$} & 0 & 0.44 \\
$O_{\text {spine }}$ & {$[\mathrm{rad}]$} & 0 & 0.22 \\
$\phi_{\text {spine }}$ & {$[\mathrm{rad}]$} & 0 & 3.14
\end{tabular}

3) Experimental setup: We conducted all experiments with Bobcat-robot on a "catwalk", a $4 \mathrm{~m}$ by $0.6 \mathrm{~m}$ wooden walkway (Fig. 4). A IR marker based motion capture (MOCAP) setup composed of twelve cameras [19], mounted around the catwalk captured the robot's absolute position over time, at $f=$ $240 \mathrm{~Hz}$. Three IR reflective markers were fixed to the front of the robot, this rigid body was tracked by the MOCAP system. MOCAP data was cleaned manually for switched

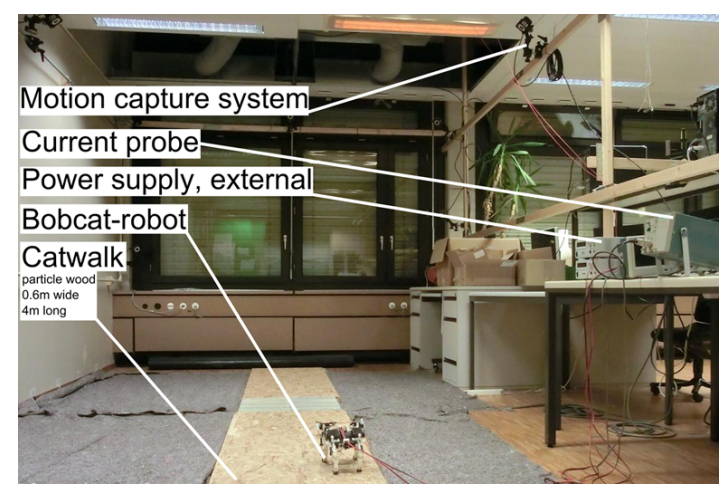

Fig. 4: Experimental setup for runs with Bobcat-robot.

marker recordings, and low pass filtered at $f=20 \mathrm{~Hz}$ in Matlab [20, R2009b]. We recorded the position of the robot, and selected a minimum locomotion distance of $1.5 \mathrm{~m}$ (eight or more locomotion cycles) to calculate the robot's average forward locomotion speed. Further, the robot's range of pitching motion was extracted. Range of pitching motion was calculated by recording the pitching angle of the robot (rotation in the robot's sagittal layer), calculating the maximum difference in a time window of the size of one locomotion cycle $(t=1 / f)$, and extracting the median of all measured values. A current probe clamped around the power tether measured the robot's power consumption. A digital oscilloscope (LeCroy 6100) sampled the amplifier output (Tektronix TM502A, AM $5033)$ at $f=100 \mathrm{kHz}$. Data was stored digitally, and low-pass filtered at $15 \mathrm{~Hz}$, in Matlab. Voltage of the power supply was directly read with a voltmeter. Standby power consumption of the RB110 control board (4 W) was subtracted from measurements. In all experiments, the robot was powered via a tether, attached to its rear end. The tether also functioned as safety cable, to pull up the robot in case it was falling, e.g. forwards. Currently, Bobcat-robot has no controller or active joint implemented to steer sideways. Hence, if a robot gait parameter combination turned the direction of the robot, the tether cable was used to carefully correct this, without reducing the robot speed. Often, the faster the robot bounding became, the more stably it was bounding in a straight line. As a general rule, the power tether was kept slack, to ensure as much as possible unconstrained robot bounding experiments. We applied four different regimes, presented as a combination of range of motor Voltage, spine amplitudes, and spine offsets (Tab. II).

\section{Results And AnAlysis}

1) From rigid spine to active spine: All experiment were divided into four categories, see Tab. II for the corresponding parameter spaces. Within the first category (rigid spine) the robot spine was locked. Corresponding experiments were based on a grid parameter testing (systematic, iterative testing) over frequency, hip amplitude, and duty factor.

$$
\begin{aligned}
\text { Frequency } f & =\{2,2.5,3,3.5\}[\mathrm{Hz}] \\
\text { Amplitude } A_{\text {hip }} & =\{0.5,0.625,0.75,0.875,1\}[\mathrm{rad}] \\
\text { Duty factor } D_{\text {vir }} & =\{0.35,0.41,0.48,0.55\}
\end{aligned}
$$

Cartesian product of above parameter sets results in 80 gaits. Other CPG control parameters were manually adjusted for each parameter combination, to reach a fast-as-possible, and 


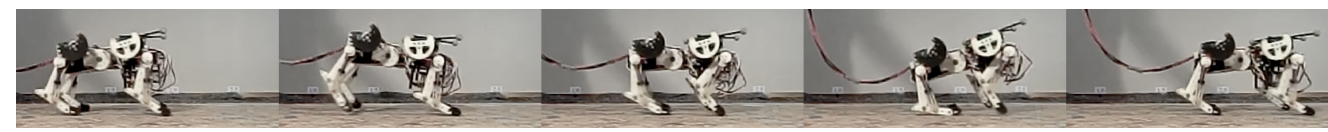

(a) Snapshot series of Bobcat-robot bounding with a fixed spine joint. This gait accelerated the robot to $0.62 \mathrm{~m} \mathrm{~s}^{-1}$. Gait cycle frequency is $3.5 \mathrm{~Hz}$.

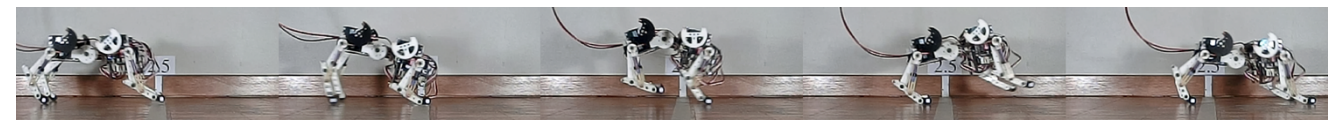

(b) Snapshot series of Bobcat-robot bounding, spine amplitude 30 degree, robot speed $0.72 \mathrm{~m} \mathrm{~s}^{-1}$, gait cycle frequency $4 \mathrm{~Hz}$.
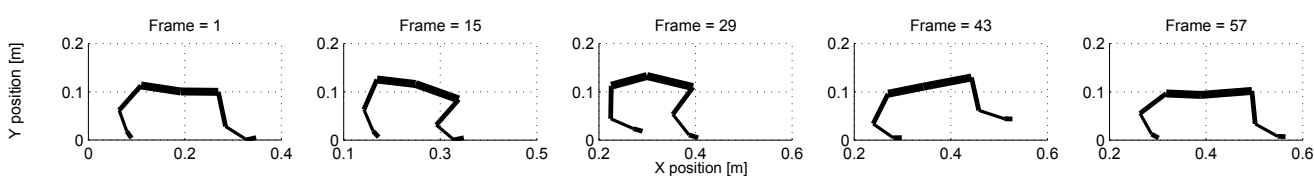

(c) Stick figure presentation of above gait, projection of the sagittal layer. Time per frame: $1 / 240 \mathrm{~s}$.

Fig. 5: Snapshots of one cycle, for fixed (a) and active (b) spine. The fixed spine gait showed much less foot clearance, applied sliding motions forward, and its hind mid-leg joint touched the ground. The active spine gait applied clear foot-clearance.
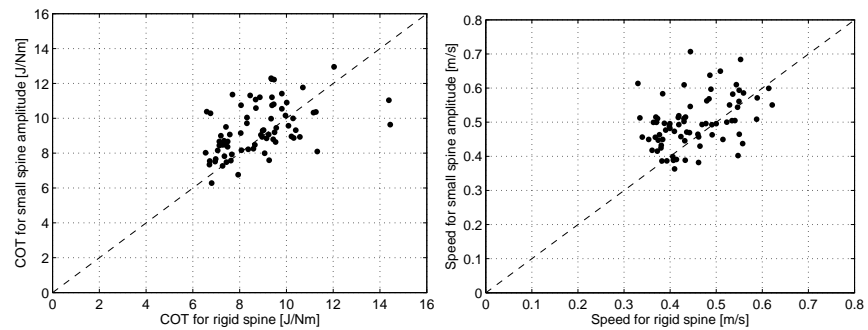

Fig. 6: Comparison of (left) COT and (right) speed, for rigid spine and small amplitude spine gaits. Each data pair applied identical main CPG parameters, but differed concerning its spine actuation.

robust gait. For higher frequency runs $(3.5 \mathrm{~Hz})$ we increased RC servo voltage from 8 to 9 Volts, to compensate for saturation effects on servo amplitudes.

Fastest gait for the rigid spine category showed an average horizontal robot speed of $0.62 \mathrm{~m} \mathrm{~s}^{-1}$ (Fig. 7), at a cost of transport $\left(\mathrm{COT}=P_{\text {electrical }} /(\mathrm{mgv})\right)$ of $8.1 \mathrm{JN}^{-1} \mathrm{~m}^{-1}$. High speed video snapshots of the best rigid spine gait $(v=$ $0.62 \mathrm{~m} \mathrm{~s}^{-1}$ ) are provided in Fig. $5(\mathrm{a}), \mathrm{CPG}$ parameters of the gait are provided in Tab. III, instantaneous speed components are plotted in Fig. 11a (thin lines). Although relatively fast speed gaits were observed (3.7 body lengths per second), such fixed-spine gaits were not fully dynamic. Robot legs showed only little ground clearance, there was almost no visible flight phase, and the robot strongly made use of forward sliding movements to propel. Gaits of the second category (small spine amplitude) applied identical main leg CPG parameters, from fixed spine gaits. However, for each set of frequency and amplitude, the best duty factor was selected and four different types of spine activity (amplitude and offset) were recorded, in total 80 gaits. Above spine activities were a combination of two spine amplitudes $\left(7.5^{\circ}, 15^{\circ}\right)$ and two offsets, bending the spine either upwards and downwards, or only only upwards. As both categories applied equal parameters, we can compare both (Fig. 6). Fig. 6b shows that the robot gained higher maximum speed when controlled with a small spine amplitude compared to a fixed spine, more than half of the gaits $(66 \%)$ are faster. Fig. 6a shows that, in average, COT for actuated spine gaits was higher, compared to rigid spine gaits $(66 \%$ of tests). COT-Speed results for all four gait categories are illustrated in Fig. 7. Comparing rigid spine and any amplitude spine gaits, we can identify that an actuated spine moves COT-vs-speed plots to the up and right, that is to higher speed, but at the cost of a higher COT. Rigid spine gaits applied the lowest COT values, up to a robot speed of $0.55 \mathrm{~m} \mathrm{~s}^{-1}$, keeping in mind that only eight out of nine motors were powered for those gaits.

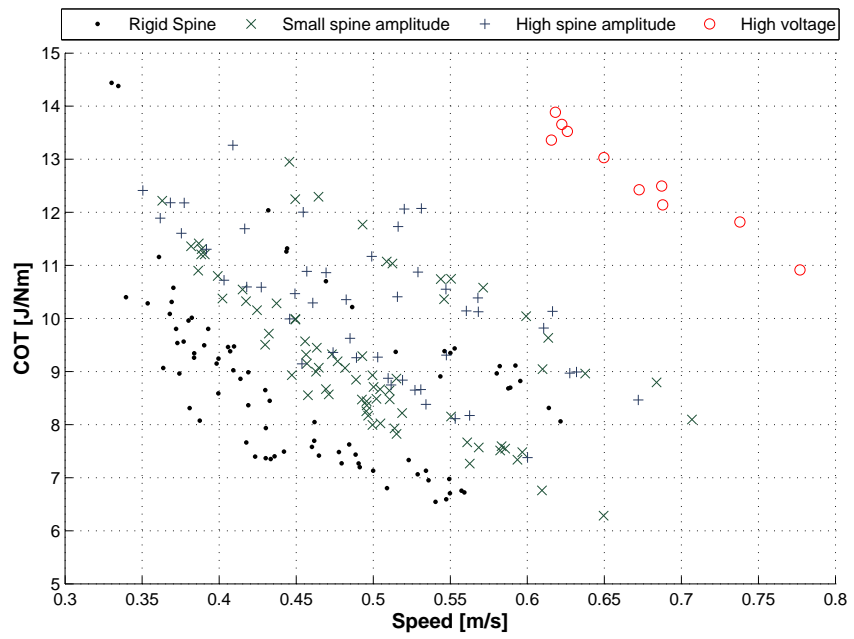

Fig. 7: COT vs. speed, from four different categories. Higher voltage moves gaits towards higher speed and COT .

2) Best spine activity: With four different spine activities within the second gait category, we were searching for the best spine amplitude and offset combination (Fig. 8). Two-side spine bending with Bobcat-robot did resulted in faster gaits, but at a higher COT. [21] hinted that a flexible spine extends hind leg length, into a longer, virtual leg (Fig. 2). Hence, both hind hip amplitude and spine amplitude work additive, less hind hip amplitude should be sufficient to gain equal or better robot speed. Hence, a higher spine amplitude in combination with relatively low hind hip amplitudes were implemented in 

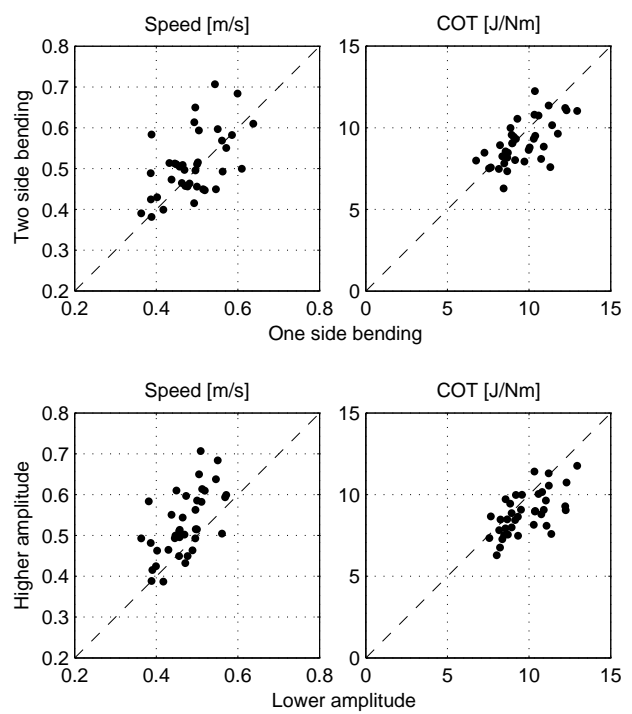

Fig. 8: Effects of spine amplitude and its curvature on speed and cost of transport (COT).

the remaining two gait categories high amplitude gait and high voltage gait.

3) High spine amplitude gaits: Based on previous results from the first and second gait category, gaits with high spine amplitude were prepared, and 50 gaits were systematically tested. Fig. 7 shows that the COT of the resulting gaits increase. However, the range of robot pitch movements was up to $2 / 3$ lower (Fig. 10), and high spine amplitude gaits showed better straight line locomotion behaviour, compared to fixed spine and low amplitude spine gaits. This improved robot stability enabled us to apply an even higher locomotion frequency $(f=4 \mathrm{~Hz})$ for the following high voltage (and high spine) amplitude experimental category.

4) High Voltage/High spine amplitude gaits: Stable and high spine amplitude gaits (up to $25^{\circ}$ ) were found within above, 3rd gait category. In the fourth category, we applied high locomotion frequency (up to $4 \mathrm{~Hz}$ ) and voltage (up to $12 \mathrm{~V}$ ) for faster oscillatory motion. Recordings of speed-COT and speed-range of pitch angle are provided in Fig. 7 and Fig. 10, respectively. The fastest gait of all 222 runs was found $\left(0.78 \mathrm{~m} \mathrm{~s}^{-1}, 4.7\right.$ body lengths per second). Snapshots and stick figure plots of a characteristically similar run $\left(v=0.72 \mathrm{~m} \mathrm{~s}^{-1}\right)$ are given in Fig. 5. Its gait diagram (Fig. 11b) shows short, but distinct flight phases, and short double stance phase sharing touchdown of front and hind limbs. Maximum spine upward curvature occurred during the flight phase. Fig. 11a shows that the range of the instantaneous vertical speed for this gait is higher than its forward component.

\section{Discussion and Future Work}

For the rigid spine configuration, we found gaits up to a robot speed of $0.62 \mathrm{~m} \mathrm{~s}^{-1}$ (3.7 body lengths per second, $\frac{\mathrm{BL}}{\mathrm{s}}$, Froude number $\left.\mathrm{FR}=\frac{v^{2}}{g l}=0.31\right)$, and the best active spine gait showed a speed of $0.78 \mathrm{~m} \mathrm{~s}^{-1}\left(4.7 \frac{\mathrm{BL}}{\mathrm{s}}, \mathrm{FR}=0.5\right.$.). Reported maximum speed for a quadruped robot with fixed spine, of roughly comparable size, weight, and actuation (Puppy1 and Puppy2, [6], [8]) is around $v=2.9 \frac{\mathrm{BL}}{\mathrm{s}}$ and $v=3.5 \frac{\mathrm{BL}}{\mathrm{s}}$.

Although our CPG network already reduced necessary control parameters to a minimum, and only direct control

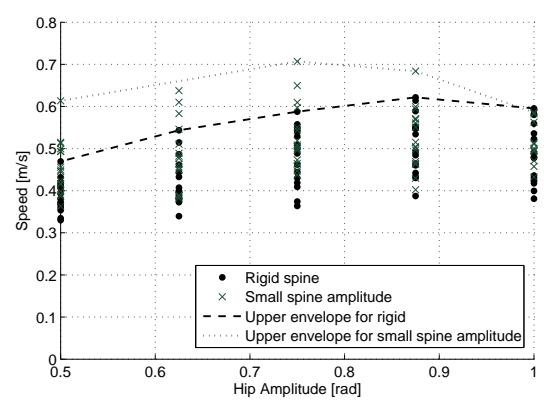

Fig. 9: Effect of hip amplitude on speed, rigid and active spine.

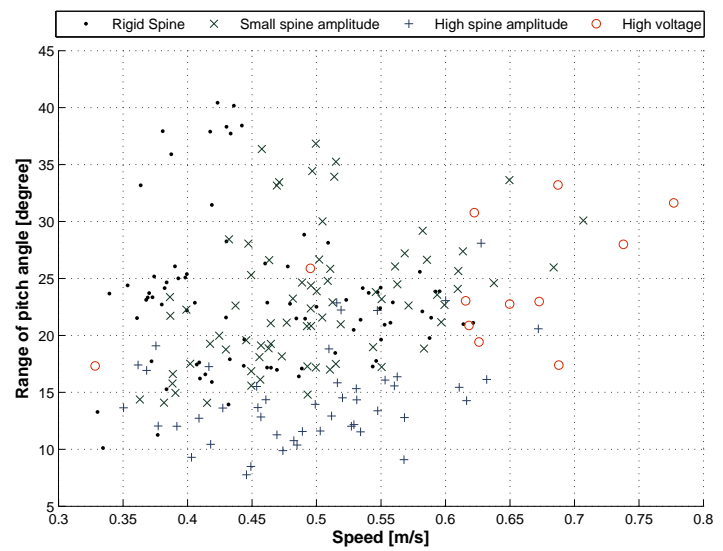

Fig. 10: Range of pitch angle vs. robot speed.

parameters were tweaked, the resulting gait-CPG parameter space is still very large. Hence, we can assume that our systematic testing only swept a limited, but as good results show, meaningful parameter space. For the future, multiple options exist to gait patterns: (a) using feedback signals, e.g. reflex-like CPG feedback [2], (b) using model based control and feedback, by simulating the robot, and designing a dedicated closed-loop controller, and (c) using optimization methods tweaking control parameters [22], or adaptive CPG parameters [23].

Confirming our first hypothesis, we could find gaits with higher speed applying active spine movement. The best found spine movement (hypothesis 2) was an upwards-downwards movement (control offset $0^{\circ}$ ), with large amplitude. This is

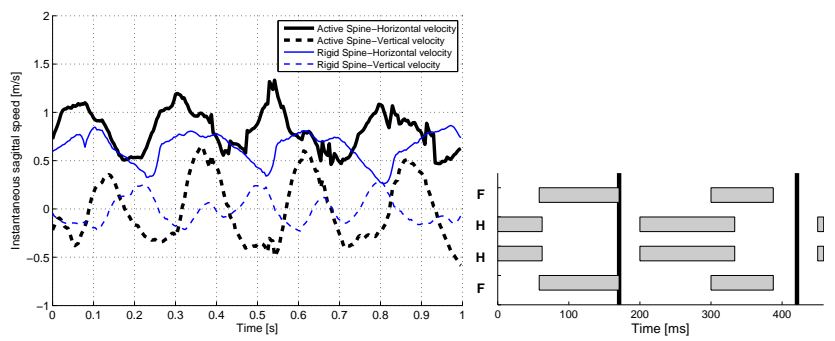

Fig. 11: (a)/left: plot of instantaneous speed (forward and vertical) for two fast gaits for fixed spine, and active spine with high motor voltage. Forward speed (continuous line) average of the rigid spine gait is $0.62 \mathrm{~m} \mathrm{~s}^{-1}$, of the high voltage, high amplitude gait $0.72 \mathrm{~m} \mathrm{~s}^{-1}$. (b)/right: gait diagram of active spine gait at $v=0.72 \mathrm{~m} \mathrm{~s}^{-1}$, data from run at Fig. $5 \mathrm{~b}$. Vertical lines mark maximum upward spine curvature. 
coherent with our second hypothesis, and can be a indicator for future passive-active spine implementations.

However, the advantage in stride length per cycle is currently not strong, a $10 \%$ higher stride length for the active spine was observed (best rigid spine: $0.177 \mathrm{~m}$, best active spine: $0.195 \mathrm{~m}$ ). At the same time, the best active spine gait applied less hip amplitude activation (Fig. 9), this indicates that higher stride lengths are possible, more analysis of current gait patterns will be necessary. In animals (and BostonDynamics Cheetah robot) overstepping of hind feet over front feet is visible for high speed gaits, with Bobcat-robot this was not reached. However, good flight phases and very little sliding was visible at the best active spine gaits, this was a large improvement to rigid-spine gaits, where the robot made use of forward sliding motions to propel. Generally, the active spine joint improved locomotion robustness, and allowed for higher locomotion frequency runs, which were not possible with a fixed spine.

In terms of cost of transport (COT) all found gaits are above $6 \mathrm{JN}^{-1} \mathrm{~m}^{-1}$, and typically no energy advantage was visible when using the active spine. An in-series elasticity could lower COT of high-voltage, high spine amplitude gaits. However, current COT is largely a product of the extremely high gear ratio of the RC servo motors (around $300: 1$ ). Adapted gearbox values would be necessary to lower COT significantly.

\section{Conclusion}

We present our approach to control active spine movements of a small bounding, quadruped robot with two-segment, compliant legs, and compare gaits with the robot in the rigid spine configuration versus the actively controlled spine configuration. Best gaits with the bounding robot with the rigid spine configuration reached a robot speed of $0.62 \mathrm{~m} \mathrm{~s}^{-1}\left(3.7 \frac{\mathrm{BL}}{\mathrm{s}}\right.$, $\mathrm{FR}=0.31)$ at a cost of transport $\mathrm{COT}=6.5 \mathrm{JN}^{-1} \mathrm{~m}^{-1}$. Best active spine gaits reached $0.78 \mathrm{~m} \mathrm{~s}^{-1}\left(4.7 \frac{\mathrm{BL}}{\mathrm{s}}, \mathrm{FR}=0.5\right.$, $\left.\mathrm{COT}=10.9 \mathrm{JN}^{-1} \mathrm{~m}^{-1}\right)$. Active spine gaits showed distinct flight phases, double-leg stance phases, and more stable gait patterns than rigid spine gaits. Here, we considered two gait features for a higher stability: a) less sliding of the feet on the ground, and b) a higher directional stability. Gaits with fixed spine often let the robot turn on the spot or change direction, while we observed that an appropriate spine actuation led to more straight-forward gaits. Further, comparing gaits of equal speed from rigid versus active spine, active spine gaits showed less pitching in the anterior robot spine (front).

\section{ACKNOWLEDGMENT}

We thank Peter Eckert for assisting experiments, and Massimo Vespignani for assisting robot assembly. Research leading to these results has received funding from the European Community's Seventh Framework Programme FP7/20072013-Challenge 2-Cognitive Systems, Interaction, Roboticsunder grant agreement No. 248311 (AMARSi).

\section{REFERENCES}

[1] N. Kohl and P. Stone, "Policy gradient reinforcement learning for fast quadrupedal locomotion," in 2004 IEEE International Conference on Robotics and Automation, 2004. Proceedings. ICRA '04, vol. 3. IEEE, 2004, pp. 2619- 2624 Vol.3.

[2] H. Kimura, Y. Fukuoka, and A. Cohen, "Adaptive dynamic walking of a quadruped robot on natural ground based on biological concepts," The International Journal of Robotics Research, vol. 26, no. 5, pp. 475-490, 2007.
[3] S. Rutishauser, A. Spröwitz, L. Righetti, and A. J. Ijspeert, "Passive compliant quadruped robot using central pattern generators for locomotion control," in 2008 IEEE International Conference on Biomedical Robotics and Biomechatronics, 2008.

[4] M. Raibert, "Trotting, pacing and bounding by a quadruped robot," Journal of Biomechanics, vol. 23, no. Supplement 1, pp. $79-81,1990$.

[5] C. Semini, N. G. Tsagarakis, E. Guglielmino, M. Focchi, F. Cannella, and D. G. Caldwell, "Design of hyq - a hydraulically and electrically actuated quadruped robot," IMechE, Part I: Journal of Systems and Control Engineering, vol. 225, no. 6 , pp. $831-849,2011$.

[6] F. Iida and R. Pfeifer, "Cheap rapid locomotion of a quadruped robot: Self-stabilization of bounding gait," Intelligent Autonomous Systems, 2004.

[7] I. Poulakakis, J. A. Smith, and M. Buehler, "Modeling and experiments of untethered quadrupedal running with a bounding gait: The scout II robot," The International Journal of Robotics Research, vol. 24, no. 4, pp. 239-256, 2005.

[8] F. Iida, G. Gomez, and R. Pfeifer, "Exploiting body dynamics for controlling a running quadruped robot," in , 12th International Conference on Advanced Robotics, 2005. ICAR '05. Proceedings. IEEE, 2005, pp. 229-235.

[9] M. Raibert, K. Blankespoor, G. Nelson, and R. Playter, "BigDog, the Rough-Terrain quadruped robot," in Proceedings of the 17th IFAC World Congress, COEX, South Korea, 2008, pp. $10823-10825$.

[10] Z. G. Zhang and H. Kimura, "Rush: a simple and autonomous quadruped running robot," Proceedings of the Institution of Mechanical Engineers, Part I: Journal of Systems and Control Engineering, vol. 223, no. 3, pp. 323-336, 2009.

[11] J. A. Smith, I. Poulakakis, M. Trentini, and I. Sharf, "Bounding with active wheels and liftoff angle velocity adjustment," The International Journal of Robotics Research, vol. 29, no. 4, pp. $414-427,2010$.

[12] M. S. Fischer, N. Schilling, M. Schmidt, D. Haarhaus, and H. Witte, "Basic limb kinematics of small therian mammals," J Exp Biol, vol. 205, no. 9, pp. 1315-1338, May 2002.

[13] M. Kani, M. Derafshian, H. Bidgoly, and M. Ahmadabadi, "Effect of flexible spine on stability of a passive quadruped robot: Experimental results," in Robotics and Biomimetics (ROBIO), 2011 IEEE International Conference on, Dec. 2011, pp. $2793-2798$.

[14] Boston Dynamics, "BD Webpage, webpage."

[15] V. A. Tucker, "Energetic cost of locomotion in animals," Comparative Biochemistry and Physiology, vol. 34, no. 4, pp. 841-846, Jun. 1970.

[16] A. Kuo, "Choosing your steps carefully," Robotics 83 Automation Magazine, IEEE, vol. 14, no. 2, pp. 18-29, 2007.

[17] A. Spröwitz, A. Tuleu, M. Vespignani, M. Ajallooeian, E. Badri, and A. Ijspeert, "'towards dynamic trot gait locomotion-design, control, and experiments with cheetahcub, a compliant quadruped robot"," 2013, accepted for publication, IJRR.

[18] A. A. Biewener, "Allometry of quadrupedal locomotion: The scaling of duty factor, bone curvature and limb orientation to body size," Journal of Experimental Biology, vol. 105, no. 1, pp. $147-171,1983$.

[19] Naturalpoint, Inc., "Optitrack s250e," 2011.

[20] T. MathWorks, "Mathworks."

[21] H. Witte, J. Biltzinger, R. Hackert, N. Schilling, M. Schmidt, C. Reich, and M. Fischer, "Torque patterns of the limbs of small therian mammals during locomotion on flat ground," $J$ Exp Biol, vol. 205, pp. 1339-1353, 2002.

[22] H. J. Bidgoly, A. Vafaei, A. Sadeghi, and M. N. Ahmadabadi, "Learning approach to study effect of flexible spine on running behavior of a quadruped robot." World Scientific Publishing Co. Pte. Ltd., 2010, pp. 1195-1201.

[23] L. Righetti, J. Buchli, and A. J. Ijspeert, "Adaptive Frequency Oscillators and Applications," The Open Cybernetics and Systemics Journal, vol. 3, pp. 64-69, 2009. 